\title{
A Retrospective Study of Granulomatous Lesions of the Larynx: Decision-making in Malignant Mimickers
}

\author{
Nupur K Nerurkar ${ }^{1} \odot$, Shruti Dhingra ${ }^{2} \odot$, Ranjita $\mathrm{KM}^{3}$
}

\begin{abstract}
Granulomatous conditions of the larynx constitute a heterogeneous group of lesions with overlapping symptoms. Many laryngoscopy findings are nonspecific, often confusing, with few systemic manifestations, and sometimes present as acute airway emergencies. It is imperative that the laryngologist suspects the possibility of such lesions when the presentation is atypical and the diagnosis does not "fit" in the classical scheme. Most cases may be managed conservatively avoiding an unnecessary tracheostomy. The correct approach undertaken at this time is crucial in determining the prognosis of the patient. An effective strategy involves multidisciplinary teams and a keen eye for systemic features, before undertaking any surgical intervention.

Most granulomatous diseases show a definite regional preponderance but with the increase in immunocompromised conditions and globalization, this trend is changing worldwide.

We retrospectively reviewed the data of 252 patients with suspected granulomatous lesions, seen over an 8-year period (2012-2020) at a tertiary care hospital with a voice clinic, in India. The epidemiological details, stroboscopy findings, histopathology, and response to treatment were noted. Some of the interesting cases with atypical presentations, which posed a diagnostic challenge, are discussed.

Keywords: Granulomatous lesions, Hoarseness, Larynx, Subglottic stenosis.

International Journal of Phonosurgery \& Laryngology (2021): 10.5005/jp-journals-10023-1225
\end{abstract}

\section{INTRODUCTION}

The prevalence of granulomatous lesions of the larynx varies in different parts of the world. They have a broad clinical spectrum and presentations. Often clinical features overlap and laryngoscopy findings do not fit the classical book description. Many of these lesions either coexist or mimic more sinister pathologies such as malignancy, leading to multiple surgical interventions. A thorough examination is imperative to rule out multisystem involvement and give a clue to the diagnosis. Delay results in irreversible changes in the laryngeal and tracheal morphology with severe patient debilitation.

Aim of this study was to review the data of 252 patients who were clinically suspected to have granulomatous lesions and to analyze how many of these were truly granulomatous either on histopathology or based on the response to treatment.

\section{Materials and Methods}

This was a retrospective clinical case study conducted in the Laryngology unit at Bombay Hospital, Mumbai, over a span of 8 years from 2012-2020. The charts of these patients were reviewed and epidemiological details, stroboscopy findings, histopathology, and response to treatment was studied.

\section{Results with Case Descriptions}

Of the 252 suspected cases, 197 were proven to be granulomatous lesions (78.1\%). Of these 114 suspected fungal (candida) laryngitis, all were correctly diagnosed. A total of 35 laryngeal tuberculosis, 28 amyloidosis, 14 granulomatosis with polyangiitis (GPA), 4 sarcoidosis, and 2 rare fungi (aspergillus and mucormycosis) were diagnosed histopathologically (Fig. 1). Of these, 22.3\% (44 patients) presented with acute airway emergency, $6 \%$ (12 patients) presented with severe dyspnea on exersion, and the rest $71.5 \%$ (141 patients)

\begin{abstract}
1,3 Bombay Hospital Voice and Swallowing Centre, Bombay Hospital and Medical Research Centre, Mumbai, Maharashtra India

${ }^{2}$ Department of Otolaryngology and Head \& Neck Surgery, Maulana Azad Medical College and Lok Nayak Jai Prakash Narayan Hospital, New Delhi, India

Corresponding Author:Shruti Dhingra, Department of Otolaryngology and Head \& Neck Surgery, Maulana Azad Medical College and Lok Nayak Jai Prakash Narayan Hospital, New Delhi, India, Phone: +919868453871, e-mail: shrudoc@hotmail.com

How to cite this article: Nerurkar NK, Dhingra S, Ranjita KM. A Retrospective Study of Granulomatous Lesions of the Larynx: Decision-making in Malignant Mimickers. Int J Phonosurg Laryngol 2021;11(2):39-45.

\section{Source of support: Nil}

Conflict of interest: None
\end{abstract}

presented with voice change. Through this study, we discuss a few of the interesting cases that had atypical presentations and overlapping signs.

\section{Fungal Laryngitis}

We reviewed 116 patients of fungal laryngitis $(\mathrm{FL})$ over a period of 8 years. The average age of presentation was 21-66 years (mean 43 years) and the duration of hoarseness lasted 1-3 months. Candidiasis was the most common infection seen in 114 cases and there was one case each of aspergillosis and mucormycosis. The appearance in these 114 patients was typical with cheesy, flaky white lesions with surrounding erythema over the vocal folds. Barring 1, all patients had isolated fungal laryngitis with no oropharyngeal or other organ involvement and $58 \%$ of these patients were immunocompetent.

We discuss one patient in our series, who had an extremely atypical presentation with pulmonary and laryngeal mucormycosis. 


\section{CASE 1}

A 51-year-old uncontrolled diabetic and hypertensive patient presented to the chest physician and was diagnosed with pulmonary mucormycosis. He was referred to the voice clinic with a breathy voice, dyspnea on exertion, and difficulty in swallowing with nasogastric feeding since the past 1 month. Laryngoscopy revealed crusting in the subglottis and bilateral immobile vocal folds in a paramedian position with a $3 \mathrm{~mm}$ glottic chink (Fig. 2A). Functional endoscopic evaluation of swallowing suggested a bilateral sluggish laryngeal adductor reflex but the patient had a good laryngeal elevation and following a few sessions of swallowing therapy could swallow orally without any aspiration (Fig. 2B). The pulmonologist reported edematous, polypoidal bronchial mucosa with thick, creamy white secretions. CT, histopathology, and fungal cultures from the lavage revealed diffuse, invasive, pulmonary mucormycosis (Fig. 2C). Though the pulmonary mucormycosis responded to Amphotericin $B$, the vocal folds slowly took a median position over the next couple of months necessitating a lateralization procedure for his increasing stridor. A left Kashima cordotomy was performed and the patient is currently breathing and eating well.

\section{Laryngeal Tuberculosis}

We retrospectively analyzed the data of 68 patients who were suspected to have laryngeal tuberculosis based on clinical symptoms

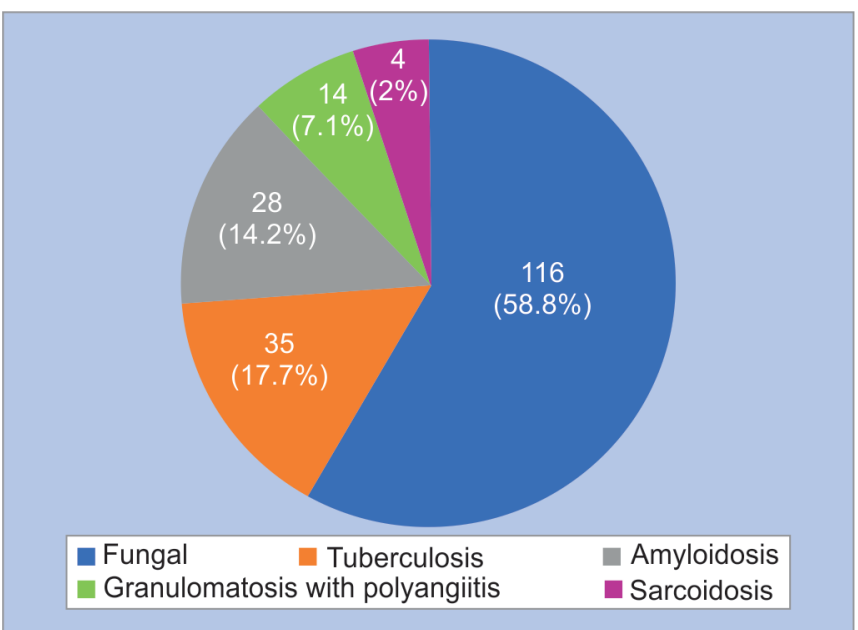

Fig. 1: Pie chart depicting distribution of granulomatous diseases of change in voice, odynophagia, and cough for more than 2 weeks with diffuse or isolated laryngeal lesions. A total of 29 patients had laryngeal manifestations secondary to pulmonary involvement with a positive sputum AFB test and radiological evidence of tuberculosis. Of the remaining 39 patients, who had three consecutive negative sputum AFB test, a normal hematological profile and no radiological evidence of disease, 20 were subjected to histopathology for a confirmatory diagnosis, while the rest (19 patients) responded well to conservative management (dietary and lifestyle modifications and proton pump inhibitors) and were excluded from the category of tuberculosis. Out of the 20 patients in whom a biopsy was taken, six patients were detected positive for primary laryngeal tuberculosis.

Secondary laryngeal tuberculosis was detected in $82.8 \%$ of our cases and they were all diagnosed correctly. However, the false positivity rate of primary laryngeal tuberculosis was high (42.4\%) and true positive cases could only be correctly diagnosed on histopathology (17.14\%). We discuss a few of the interesting cases of primary laryngeal tuberculosis.

\section{CASE 2}

A 40-year-old patient presented with hoarseness for a month. Laryngoscopy revealed an irregular growth confined to the right infraglottic region (Fig. 3A). Bilateral vocal folds were mobile but there was a decreased mucosal wave formation of the right side. She underwent excision of the posterior two-third of the lesion using an acublade $\mathrm{CO}_{2}$ laser (Fig. 3B) and histopathology was suggestive of caseating granulomas. On receiving antitubercular treatment for 6 months, good healing of the vocal folds was observed with no recurrence of the disease.

\section{CASe 3}

A 60-year-old patient presented with apparently sudden onset hoarseness with no prior history of smoking, hypothyroidism, or tuberculosis. On examination, the right vocal fold appeared inflamed, irregular, and asymmetric (Fig. 4A). There was an incomplete phonatory gap along with a reduced mucosal wave on the right side. A differential diagnosis of acute laryngitis, tuberculosis, erythroplasia, and early carcinoma was kept in consideration as the sputum was negative for AFB and the chest X-ray was clear. The patient underwent a transoral laser excision of the lesion (Fig. 4B). Histopathology revealed caseating granulomas, suggestive of primary laryngeal tuberculosis and the lesion resolved completely with AKT.

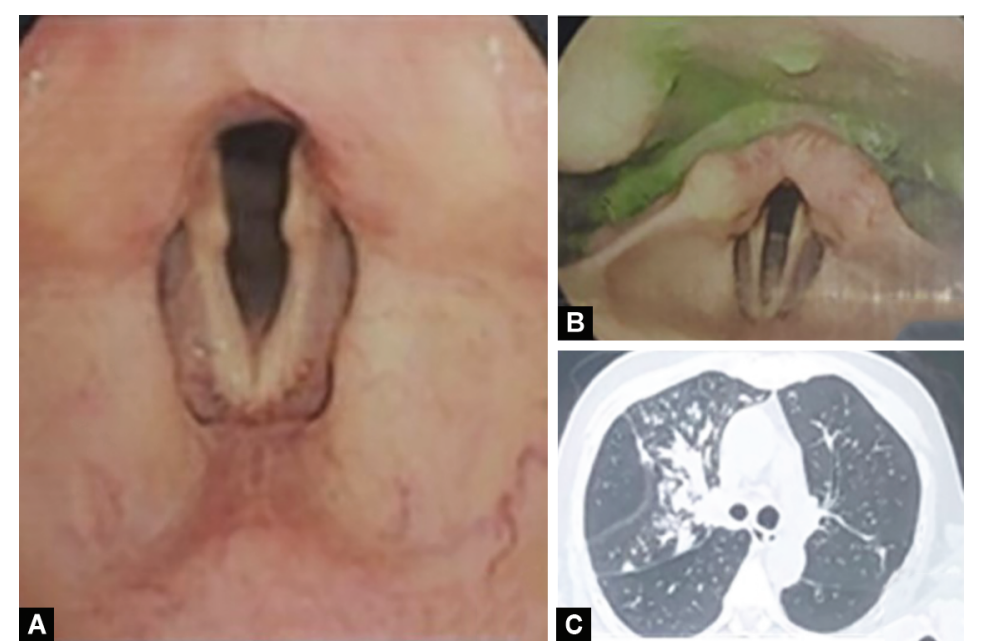

Figs $2 \mathrm{~A}$ to $\mathrm{C}$ : (A) B/L immobile vocal folds; (B) FEES following therapy revealing no aspiration of water; (C) CT of the chest revealing the enhancing lesions of pulmonary mucormycosis 

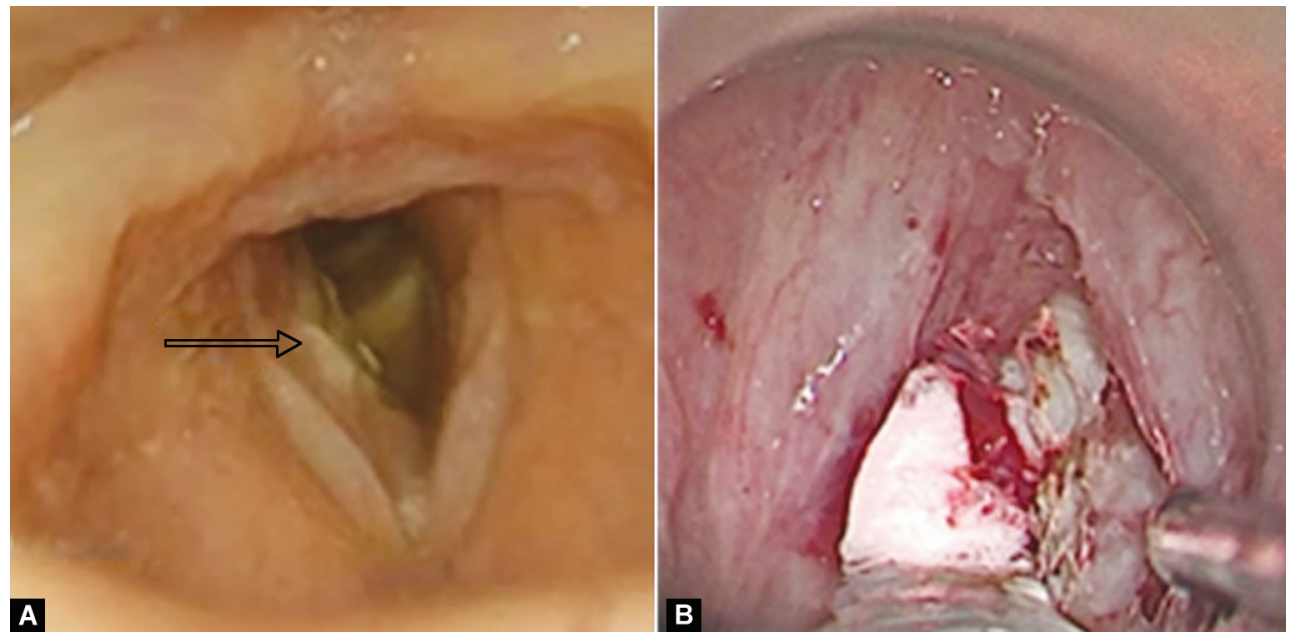

Figs 3A and B: (A) Stroboscopy revealing a right infraglottic lesion; (B) Microlaryngeal surgery revealing a cauliflower type of lesion of the right infraglottis
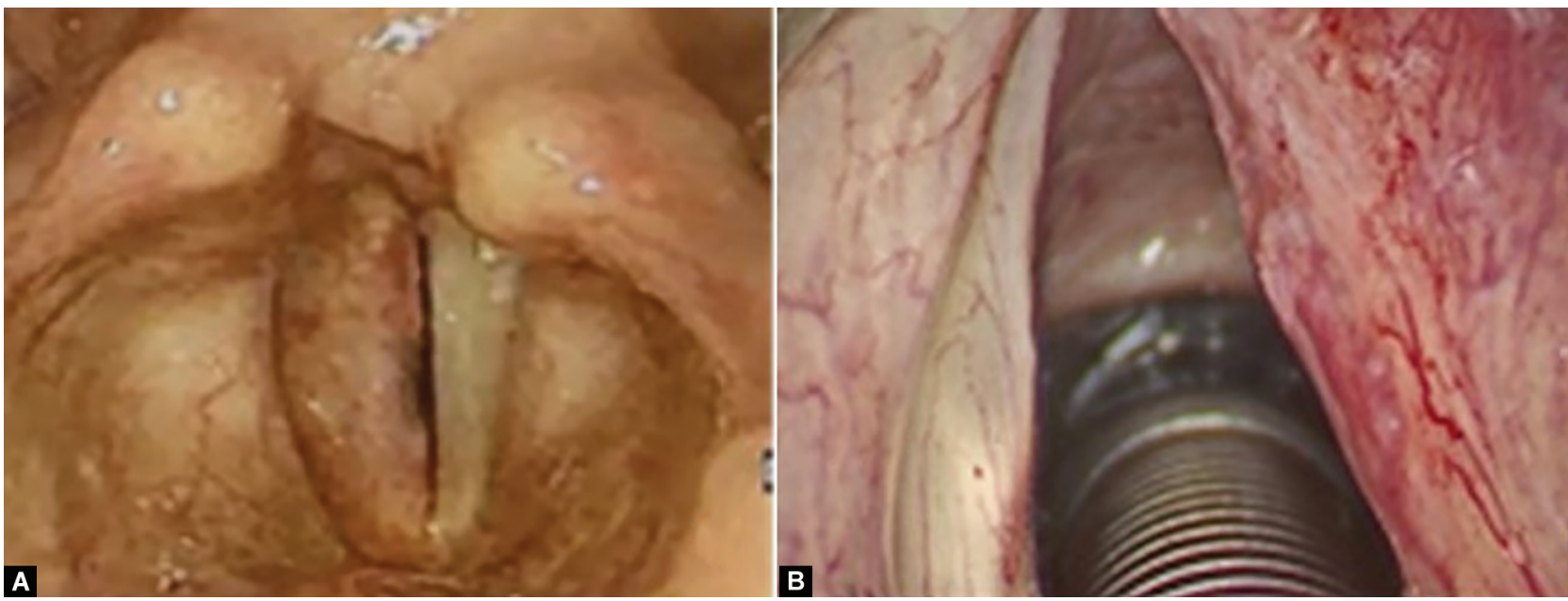

Figs 4A and B: (A) Right vocal fold congestion and irregularity observed on stroboscopy; (B) The same lesion under microscopic magnification

\section{Laryngeal Sarcoidosis}

\section{CASE 4}

A 69-year-old smoker, on chronic dialysis presented with a weak and hoarse voice for the last 8 months. Laryngeal examination revealed an irregular appearing right vocal fold with hyperemia and decreased mucosal wave (Fig. 5). There was no radiological evidence of disease in the chest. A clinical impression of early malignancy or primary tuberculosis was kept under consideration. Complete excision of the epithelial lesion was undertaken using a $\mathrm{CO}_{2}$ Acublade. The histopathology was suggestive of sarcoidosis. The patient was started on oral steroids at $30 \mathrm{mg}$ once daily for 3 months and then tapered over the next 3 months. In 3 years of follow-up the patient has been disease-free.

\section{Amyloidosis}

We studied 28 cases of amyloidosis with laryngeal involvement of which 27 had isolated involvement of the larynx, and one case had associated nasopharyngeal lesions with plasma cell dyscrasia. Of these 27 cases, 26 presented with severe dyspnea and hoarseness and had diffuse disease with amyloid deposits at multiple subsites including the false and true vocal folds, ventricles, epiglottis, and the infraglottic region, while one patient had an isolated small lesion near the left anterior commissure. We discuss two cases that presented at extremes of the clinical spectrum of laryngeal amyloidosis.

\section{Case 5}

A 50-year-old patient presented with complaints of hoarseness for the last 6 weeks. On examination, there was a small lesion on the left vocal fold, near the anterior commissure, clinically resembling a subepithelial fibrotic cyst (Fig. 6). Bilateral vocal folds were mobile with normal mucosal waves on stroboscopy. The lesion was excised using a $\mathrm{CO}_{2}$ laser under general anesthesia. Histopathology with special stains revealed subepithelial deposits of amorphous, homogenous, eosinophilic material suggestive of amyloidosis. A complete workup to rule out systemic amyloidosis was done which was negative. The patient has been on regular follow-up over the last 10 years, with a good voice, and there has been no recurrence of a lesion in the larynx or amyloid deposits detected elsewhere in the body.

\section{CASE 6}

A 62-year-old presented with hoarseness for 9 months. He was a chronic asthmatic, on inhalers, with persistent history of chest congestion, and heaviness. Laryngeal examination revealed a 


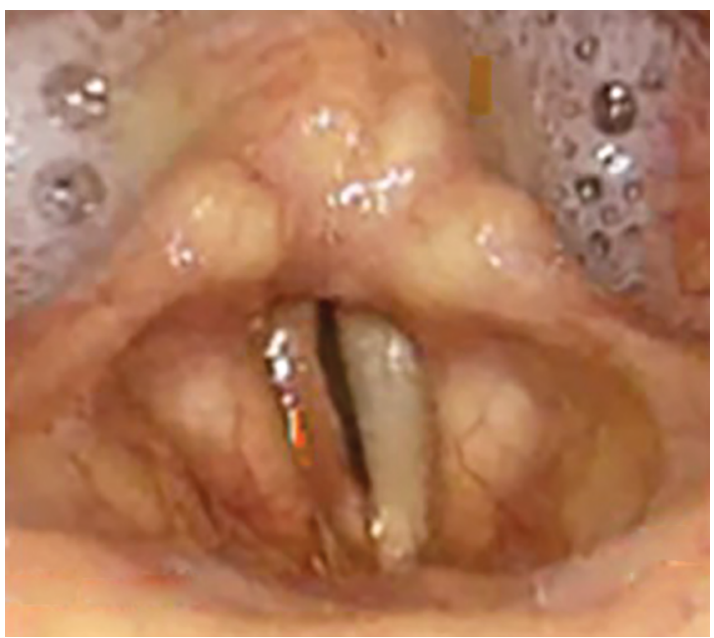

Fig. 5: Hyperemic right vocal fold

diffuse, greyish friable lesion involving the right ventricle (Fig. 7A). A deep laser incisional biopsy revealed amyloidosis with plasma cell dyscrasia. This was followed by an extensive evaluation for systemic involvement including a bone marrow biopsy to rule out multiple myeloma, which was negative. The patient received low-dose radiation therapy at another center for plasma cell dyscrasia. On 6 months of follow-up, nasal endoscopic examination revealed a friable growth in the nasopharynx (Fig. 7B), which was enhanced on the MRI scan. Biopsy was confirmatory of amyloidosis.

\section{Granulomatosis with Polyangiitis}

In our series of 14 patients, the common laryngeal symptoms were progressive hoarseness, dyspnea on exertion, and stridor necessitating tracheostomy. Subglottic stenosis was seen in all patients, nine of whom had to be tracheostomized (64.2\%). None of these patients could be decannulated over an average follow-up of 5 years. One patient presented with a diffuse infra-glottic submucosal bulge, hiding distal subglottic stenosis (which became apparent after treatment). In the remaining five patients, who did not undergo a tracheostomy, multiple endoscopic interventions were needed a minimum of six times and a maximum of nine times, (endoscopic balloon dilatation, $\mathrm{CO}_{2}$ laser resection and dilatation, and cold steel resection and dilatation) to maintain an adequate airway. Two patients also underwent open surgical procedures.

\section{Discussion}

Fungal laryngitis is usually seen as a part of generalized fungal infection involving the oral cavity and oropharynx in immunocompromised patients. Isolated involvement of the larynx in immunocompetent individuals is being seen more frequently, especially with a history of steroid intake, prolonged antibiotic use, or chronic gastroesophageal reflux. In a review of 118 patients with laryngeal candidiasis, only 13 patients (21.3\%) presented with concomitant oral candidiasis. ${ }^{1}$ Clinical features of fungal laryngitis include hoarseness, dysphonia, dysphagia, rarely pain, and dyspnea on exertion. Candida is usually the most common type (our study had $98.2 \%$ cases) and its cheesy white appearance or slough-covered ulcers may be confused with leukoplakia or acute laryngitis. Documentation of aspergillus and mucor is limited, mostly as single-case reports. In a review of 19 cases of laryngeal aspergillosis, ${ }^{2}$ none had a correct pre-operative diagnosis with differentials of tuberculosis, malignancy, lymphoma, and keratosis laryngis.

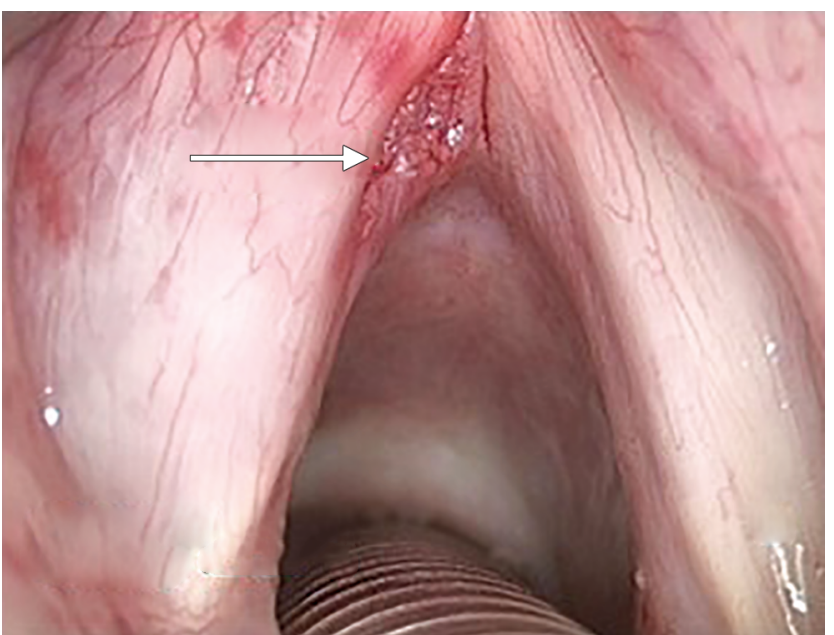

Fig. 6: Lesion resembling subepithelial fibrotic cyst on the left vocal fold near the anterior commissure

Though epiglottic edema may trigger respiratory distress, ${ }^{3}$ presence of bilateral vocal fold immobility leading to stridor is an uncommon presentation of fungal (Mucor) laryngitis. Our patient presented with dyspnea on exertion due to bilateral vocal fold immobility, without evidence of edema, slough, or irregularity on the laryngeal surface, though some crusting was evident in the subglottis.

High clinical suspicion and response to 4 weeks of anti-fungal treatment usually confirm the diagnosis. If no response is seen or there is suspicion of a malignancy, a deep biopsy after removal of slough, or rarely a laryngeal swab, with fungal microscopy and culture may be essential. A premature biopsy, however, can predispose to vocal fold scarring and should be avoided.

A dramatic response to azoles administered for 3-4 weeks in case of non-invasive and up to 3 months for invasive fungus, is seen. Frank laryngeal invasion, resistance to the prescribed antifungal or thick lesions may be refractory to treatment. One should also keep in mind the possibility of coexisting malignancy and tuberculosis with nonresponding lesions.

Laryngeal TB, though rare globally, is not uncommon in the Indian subcontinent. Besides the endemicity, an increase in the number of immunocompromised hosts, and the development of multidrug-resistant organisms, the disease is presenting itself with various nonspecific symptoms. Classical symptoms like hemoptysis, fever, weight loss, and the "turban epiglottis" are rarely seen today. ${ }^{4}$ While laryngeal involvement secondary to pulmonary kochs is more common, primary laryngeal tuberculosis accounts for less than $1 \%$ of all cases ${ }^{5}$ and is often considered with several differentials. Sputum is negative in $80 \%$ of these cases ${ }^{6}$ and CECT chest may often be misleading. ${ }^{7}$

Early stages may present only with inflammation, hyperemia, and edema over the vocal folds, ulcers, polypoidal lesions, or isolated involvement of subglottis. Diffuse asymmetric involvement of the tongue base, vallecula, and laryngeal subsites, closely resembles a malignancy, fungal infection, granulomas of Wegener's, or sarcoidosis and may present as an airway emergency. ${ }^{8}$ In our study, $42.4 \%$ cases falsely resembled primary tuberculosis and it could be correctly diagnosed only in six patients (17.14\%), after histopathology.

Sarcoidosis closely mimics tuberculosis and usually involves the epiglottis due to its rich lymphatic supply. The epiglottis may appear thickened and granular with the formation of yellowish-red nodules, "the turban epiglottis." True vocal folds are less frequently involved 

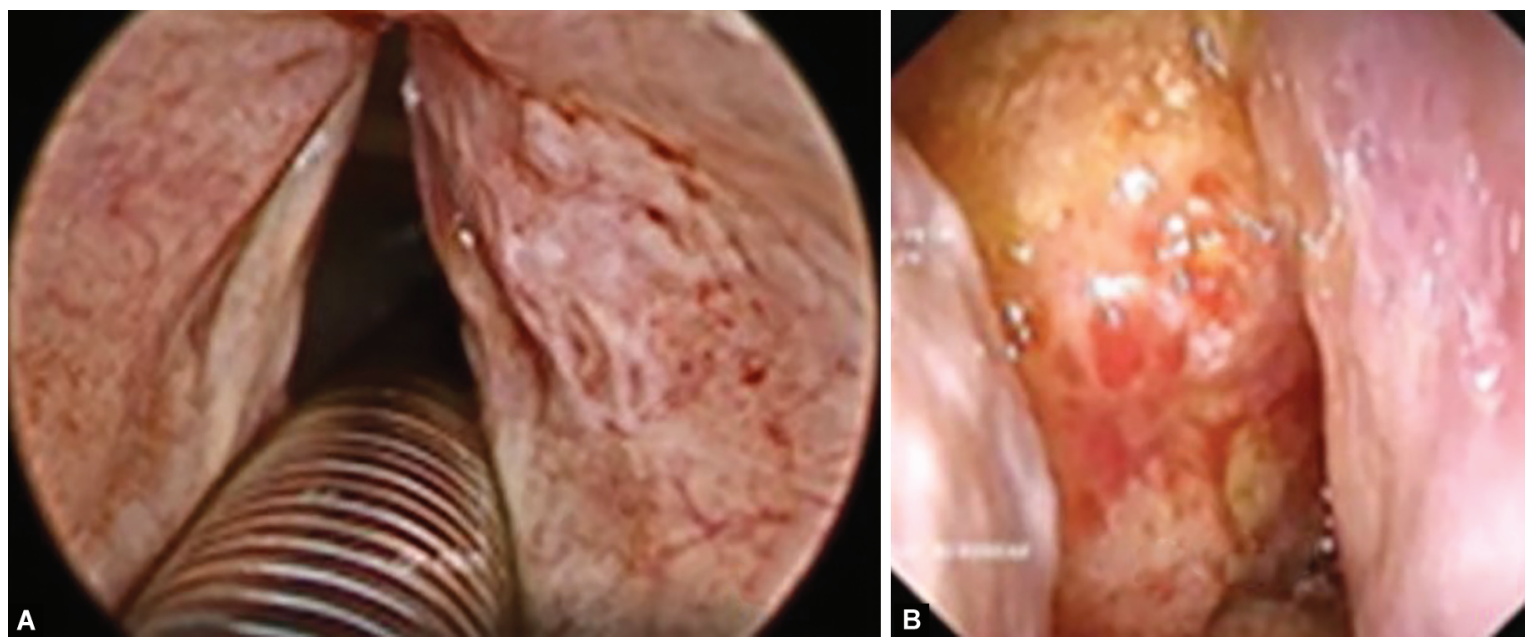

Figs 7A and B: (A) Right ventricular irregular growth; (B) Right nasopharyngeal yellowish lesion

due to the paucity of lymphatics. ${ }^{9}$ In our case, the patient presented only with hyperemia of unilateral vocal fold that was initially suspected to be primary tuberculosis. Both primary tuberculosis and sarcoidosis can exist without any pulmonary lesions. PCR and QuantiFERON-TB Gold In-Tube (QFT-GIT) assay greatly improve the detection of tuberculosis and biopsy is definitive.

A high index of suspicion must be kept, as subtle findings may mislead one to undertreat a case of tuberculosis as laryngitis, start steroids for congested vocal folds (which may flare up the disease) or overtreat it as a malignancy. Biopsy may be required for nonresponders, or to exclude malignancy. Sequelae of the untreated disease include glottic and subglottic stenosis, irreversible fibrosis in superficial lamina propria, and vocal fold paralysis.

Though amyloidosis is not typically classified as a granulomatous condition, it may be worthwhile to include in the differential diagnosis as it is considered a great masquerader. Amyloidosis can have a range of presentations that may be localized or systemic. Though laryngeal involvement is rare $(0.2-1.2 \%$ of all benign tumors) ${ }^{10}$ it usually presents with hoarseness, globus sensation, hemoptysis, dysphagia, dyspnea, or stridor warranting a tracheostomy. While supraglottis remains the most common site of involvement, there may be a localized deposit mimicking a polyp, cyst, or granuloma (as was seen in our case) or a generalized involvement with diffuse soft tissue infiltration or circumferential glottic and subglottic thickening. ${ }^{11}$

Difficulty in diagnosis arises when there is only a subtle thickening of mucosa without the characteristic yellow-grey lesions. This broadens the differentials to include malignancy, tuberculosis, and granulomatosis with polyangiitis. The other extreme of the clinical spectrum is an extensive disease involving multiple organs (as discussed in our second case) such as the nasopharynx, salivary glands, paranasal sinuses, eye, oral cavity, oropharynx, tracheobronchial tree, heart, and kidney. Deposits of amorphous, homogenous, eosinophilic material on histopathology and the presence of apple-green birefringence under polarized light after Congo Red staining, help to clinch the diagnosis.

Early diagnosis provides a serviceable voice and relatively good outcomes. Surgical treatment is warranted if an amyloid bulk is causing mechanical obstruction and significant dysphonia. Typically, the disease progression stops after 7 years possibly because of exhaustion of the underlying clonal plasma cells.
Granulomatosis with polyangiitis is an uncommon condition that is characterized by the triad of (1) involvement of the upper or lower respiratory tract by necrotizing granulomas, (2) vasculitis, and (3) focal necrotizing glomerulonephritis.

When a patient presents with the classical triad and positive ANCA reports, the diagnosis is apparent. However, this is rarely the case. Presentations in the form of epiglottic edema and ulceration, cobblestone appearance of the bronchial mucosa with erythema, and luminal synechiae, often mimic TB laryngitis, tracheobronchitis, tracheobronchial amyloidosis, and sarcoidosis. On occasion, mucosal granulomas become large enough to produce a mass lesion referred to as "inflammatory pseudotumor" which can cause hemoptysis and mimic a malignancy. ${ }^{12}$

Isolated subglottic involvement is not uncommon and maybe the sole presenting feature. The average reported incidence is approximately $16-23 \% .{ }^{13,14}$ This was however seen in all our cases and dyspnea was the prime reason the patient was referred to us. Early in its course, symptoms may be absent or very subtle, often misdiagnosed as asthma or common airway diseases. Unless biopsy or ANCA tests are positive, it is important to exclude post-intubation, infectious and idiopathic causes. Subglottic stenosis may develop or progress even when the systemic disease is under remission ${ }^{13}$ as its clinical course may be totally independent of the systemic flare.

Bronchoscopic assessment is essential to evaluate changes in the mucosa, particularly the subglottic trachea. Biopsies are mostly inconclusive ${ }^{13}$ and often show nonspecific acute and chronic inflammatory changes. Computerized tomography may suggest patchy and nodular areas of consolidation and ground glass appearance with or without cavitation. Treatment must be individualized and intralesional steroid injection, cold steel instrumentation, and balloon dilatation are suggested.

Granulomatous lesions may mimic benign or malignant conditions and remain undertreated or cause unnecessary concern. Most lesions are missed unless there is a strong clinician suspicion and special stains are requested. Often they present as acute airway emergencies and it may be a challenge to choose between noninvasive ventilation and performing an emergency airway procedure. The correct approach taken at this time goes a long way in deciding the prognosis. A stepwise management protocol (Flowchart 1) can assist in narrowing the differentials. 
Flowchart 1: Differential diagnosis of suspicious granulomatous lesions

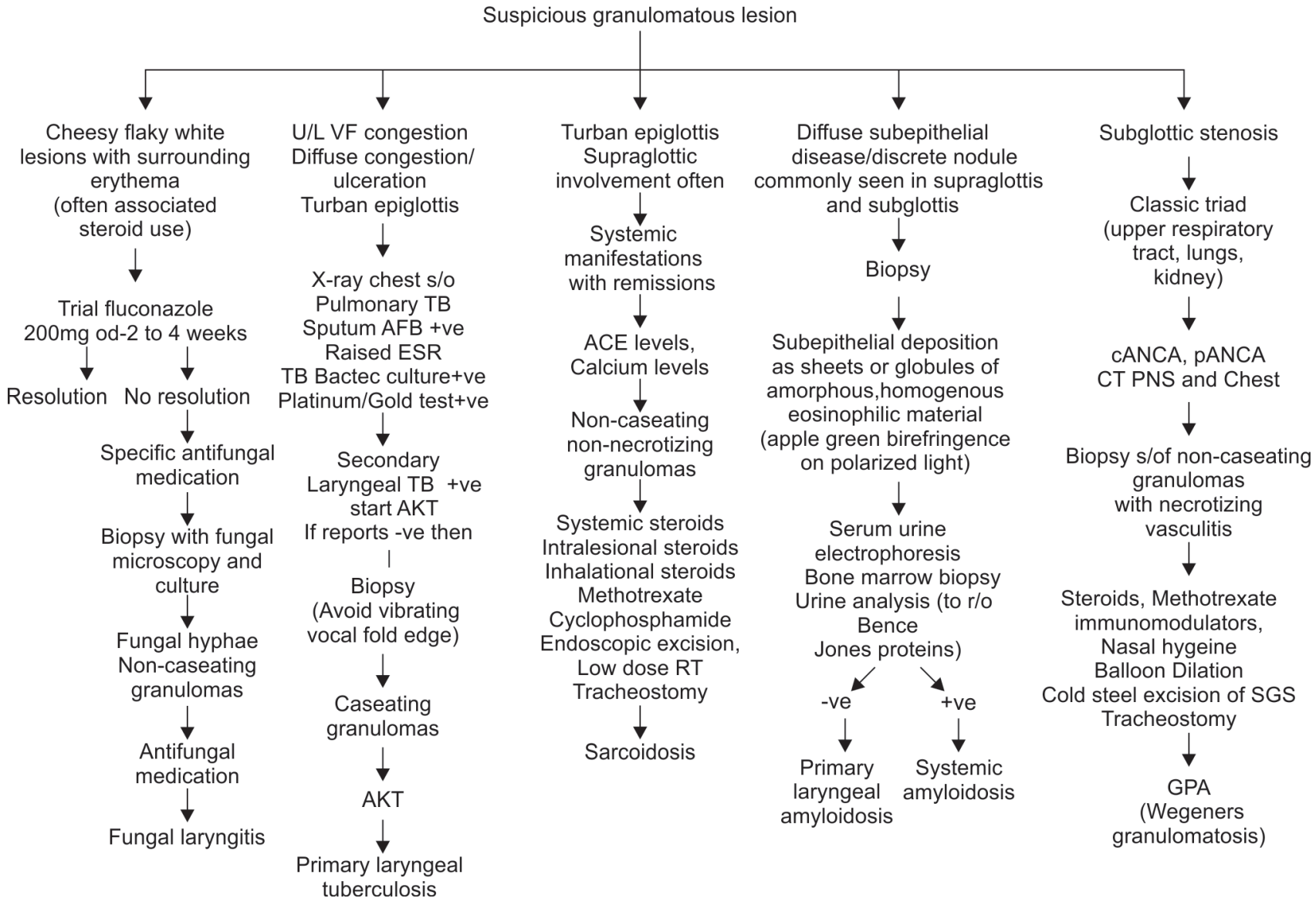

As evolution of the disease is unpredictable, with frequent flares and interventions for airway maintenance, tailoring the therapeutic strategy to suit individual patient needs constitutes a good treatment plan.

\section{Conclusion}

The larynx is seldom the initial site of involvement by granulomatous lesions and typically involvement of the chest or other regions of the head and neck often precede the laryngeal disease. Nevertheless, we need to be aware that some of these conditions may primarily involve the larynx and the findings may be subtle, with negative confirmatory reports. In our study of 252 suspected granulomatous lesions, 197 (78.1\%) lesions were accurately diagnosed as granulomatous. Lesions that closely resembled some of these (22\%) were histopathologically diagnosed to be keratosis, nonspecific chronic inflammatory tissue, severe dysplasia, and invasive squamous cell carcinoma. Therefore, it is imperative that the clinicians "think granulomatous" when the disease is a mimicker with multiple possibilities. Challenges in diagnosis may be surpassed by suspicion, a "detective's eye" and multidisciplinary teamwork.

\section{OrCID}

Nupur Kapoor Nerurkar • https://orcid.org/0000-0002-4127-1590 Shruti Dhingra $\odot$ https://orcid.org/0000-0002-0231-2310

\section{References}

1. Valente P, Ferreira J, Pinto I, et al. Management of laryngeal candidiasis: an evidence-based approach for the otolaryngologist. Eur Arch Otorhinolaryngol 2020;277(5):1267-1272. DOI: 10.1007/s00405-020-05865-4

2. Dutta M, Jotdar A, Kundu S, et al. Primary laryngeal aspergillosis in the immunocompetent state: a clinical update. Braz J Otorhinolaryngol 2017;83(2):228-234. DOI: 10.1016/j. bjorl.2015.06.002

3. Fisher EW, Richards A, Anderson G, et al. Laryngeal candidiasis: a cause of airway obstruction in the immunocompromised child. J Laryngol Otol 1992;106(2):168-170. DOI: 10.1017/s0022215100118997. PMID: 1532611.

4. Beg MHA, Marfani S. The larynx in pulmonary tuberculosis. J Laryngol Otol 1985;99(2):201-203. DOI: 10.1017/s0022215100096547

5. Uslu C, Oysu C, Uklumen B. Tuberculosis of the epiglottis: a case report. Eur Arch Otorhinolaryngol 2008;265(5):599-601. DOI: 10.1007/s00405-007-0492-9

6. Nishiike $\mathrm{S}$, Irigune $\mathrm{M}$, Kubo $\mathrm{T}$, et al. Laryngeal tuberculosis: a report of 15 cases. Ann Otol Rhinol Laryngol 2002;111(10):916-918. DOI: 10.1177/000348940211101010

7. Galli J, Nardi C, Contucci AM, . Atypical isolated epiglottic tuberculosis: a case report and a review of the literatureet al. Am J Otolaryngol 2002;23(4):237-240. DOI: 10.1053/ajot.2002.123441

8. Mehndiratta A, Bhat P, D'Costa L, . Primary tuberculosis of larynxet al. Indian J Tuberculosis 1997;44(4):211-212.

9. Devine KD. Sarcoidosis and sarcoidosis of larynx. Laryngoscope 1965;75:533-569. DOI: 10.1288/00005537-196504000-00001

10. Behnoud F, Baghbanian N. Isolated laryngeal amyloidosis. Iran J Otorhinolaryngol 2013;25(70):49-52. 
11. Almuslim HM, Alshaikh NA. Extensive laryngeal amyloidosis presenting with stridor: review of literature and case presentation. Egyptian Journal of Ear, Nose, Throat and Allied Sciences. 2014;15(1):41-44.

12. Daum TE, Specks U, Colby TV, et al. Tracheobronchial involvement in Wegener's granulomatosis. Am J Respir Crit Care Med 1995;151 (2 Pt 1):522-526. DOI: 10.1164/ajrccm.151.2.7842215
13. Gluth MB, Shinners PA, Kasperbauer JL. Subglottic stenosis associated with Wegener's Granulomatosis. Laryngoscope 2003;113(8):1304-1307. DOI: 10.1097/00005537-200308000-00008

14. O'Devaney K, Ferlito A, Hunter BC, . Wegener's granulomatosis of the head and necket al. Ann Otol Rhinol Laryngol 1998;107 (5 Pt 1):439-445. DOI: 10.1177/000348949810700515 